\title{
Changes in the frequency and pattern of drugs detected among suspected drug users during the COVID-19 pandemic in Turkey
}

\author{
Yeter Erol Öztürk ${ }^{1}\left[\right.$ Oya Yeter $^{1} \cdot$ Ismail Ateş $^{1}$
}

Received: 29 September 2021 / Accepted: 3 February 2022 / Published online: 11 February 2022

( $)$ The Author(s), under exclusive licence to Springer-Verlag GmbH Germany, part of Springer Nature 2022

\begin{abstract}
From March 13 until May 31, 2020, a complete lockdown in Turkey was planned and implemented by the government of Turkey. The vulnerable population with substance use disorders was affected more than others due to the social isolation measures meant to control the pandemic. This study presents detailed and broad data on drug abuse in suspected cases during the first wave of the COVID-19 pandemic in Turkey and compares the frequencies and patterns of drug abuse before and during the pandemic. The samples were screened by liquid chromatography-high resolution mass spectrometry and liquid chromatography-tandem mass spectrometry. Blood and urine samples of suspected users $(n=9669)$ were analyzed for drugs of abuse during the first wave of the COVID-19 pandemic and compared with their results $(n=8727)$ obtained just before the COVID-19 pandemic. The use of party drugs, such as MDMA and cocaine, and the classic illicit drug cannabis was significantly decreased and followed the same trend after complete lockdown was over. In contrast, methamphetamine use increased significantly during the lockdown period and continued after the lockdown. Interestingly, the number of tests that were positive for pregabalin as a misused licit drug increased, and this increase continued after the lockdown. The results showed a significant increase in drug abuse cases and changes in drug abuse trends, with an alteration toward more easily obtainable and lower-priced drugs. Using more dangerous and easily available licit and illicit drugs may cause serious health problems.
\end{abstract}

Keywords COVID-19 $\cdot$ Turkey $\cdot$ Drug abuse $\cdot$ Frequency $\cdot$ Pattern

\section{Introduction}

In November 2019, a new type of coronavirus (later named COVID-19) was reported in Wuhan [1]. COVID-19 was declared a pandemic on March 11, 2020 by the WHO due to the severity of the disease [2]. As of 15 September, more than 226 million people have been infected with the virus, $2.0 \%$ of whom have died worldwide [3]. Fortunately, authorized treatments and vaccines have brought new hope to the fight against the global pandemic.

Since mid-January 2020, a variety of preventive measures have been implemented in private and public areas in Turkey. On March 13, four new cases were reported, schools were closed, and some other precautions were implemented, such as performing sporting events without fans and canceling

Yeter Erol Öztürk

yetererol@hotmail.com

1 Council of Forensic Medicine, Chemistry Department, 34196, Bahcelievler, Istanbul, Turkey all professional and nonprofessional gatherings and events. Restaurants were allowed only takeout and delivery. Shopping centers, bars, gymnasiums, and mosques were closed. Citizens were asked to stay at home to keep themselves in voluntary quarantine. On March 22, a total curfew was announced for people who had chronic diseases or were over the age of 65. On April 3, the curfew was expanded to people who were twenty or younger. As of June 1, Turkey initiated a normalization process due to reported low case numbers, and most of the restrictions were canceled [4, 5]. As in many other countries, the Turkish government planned and applied for the control of the pandemic, including tracing, isolation, testing, judicial fines, restricting the movement of people across the country, and vaccinating. As of September 15, 2021, more than 6.2 million people had been infected with the virus, $0.9 \%$ of whom had died in Turkey [3].

The risks of a new wave and peak of infections continue to cause uncertainty among people around the world. The rapidly evolving situation has dramatically transformed people's lives. The consequences of COVID-19 associated 
with the virus, including inadequate supplies, interruption of social life, risk of infection and death, and fear of lockdown, can cause destructive effects in private and public life [6]. Additionally, with its rapid spread, the COVID-19 pandemic has a high death rate and causes substantial fear, panic, psychosis, anxiety, trauma, and suicide risk around the globe [7]. During a pandemic, this isolated and stressed population habitually tends to use substances to mitigate their negative feelings [8]. People with substance use disorders are more prone to be negatively affected by involuntary lockdown [8]. Although the literature is insufficient about substance use in COVID-19, previous studies suggest increases in substance use during an epidemic [9].

To the best of our knowledge, this is the first study to investigate drug abuse trends in Turkey among suspected drug users during the first year of the COVID-19 pandemic and compare the data with the last year just before the pandemic using a retrospective analysis of case results.

\section{Methods}

\section{Sample collection}

This study was conducted in accordance with the approval of the Presidency of the Scientific Board of the Council of Forensic Medicine (Approval Number:21589509/2021/747). The forensic samples that were sent to the Presidency of the Council of Forensic Medicine by legal authorities between March 1, 2019 and February 29, 2020 and between March 1, 2020 and February 28, 2021 were analyzed using routine methods used in abused drug screening protocols. In total, 8727 subjects were sampled between March 1, 2019 and February 29, 2020, and 9669 were sampled between March 1, 2020 and February 28, 2021. Blood and urine samples were used for the study and were taken from persons suspected of drug abuse. None of the blood or urine samples were taken specifically for this study. Both blood and urine samples were collected from each suspected drug abuse for analysis of measured substances. Samples were obtained from the Department of Chemistry in the Council of Forensic Medicine of the Ministry of Justice. The samples were stored at $-20{ }^{\circ} \mathrm{C}$ before analysis.

\section{Sample preparation and analysis}

Fifty microliters of internal standard mix was added to 0.5 $\mathrm{mL}$ of blood and urine samples. Five milliliters of distilled water was added to the prepared samples. The samples were centrifuged for $5 \mathrm{~min}$ at $4400 \mathrm{rpm}$ after vortexing. The samples were prepared using OASIS HLB cartridges with an LC Tech Fully Automated solid-phase extraction instrument. SPE was performed according to the methods of Ozturk et al. and Yeter et al. [10, 11]. After extraction, the samples were evaporated under a stream of nitrogen at $40{ }^{\circ} \mathrm{C}$ until fully dried and reconstituted with mobile phases $\mathrm{A}$ (water containing $5 \mathrm{mM}$ ammonium acetate and $0.1 \% \mathrm{v} / \mathrm{v}$ formic acid) and $B$ (methanol) (v:v, 80:20).

A list of abused drugs was formed according to TCK-191 (Turkish Criminal Law) [12], and routine drug abuse screenings were used to screen for controlled drugs (Table S1). In the general method, drug analysis was performed by liquid chromatography tandem mass spectrometry (LC-MS/MS), and liquid chromatography-high-resolution mass spectrometry (LC-HRMS) techniques were carried out complementarily. LC-MS/MS analyses were performed using an AB Sciex QTRAP 5500 triple quadruple linear ion trap mass spectrometer instrument, and a Restek Ultra Biphenyl (150 $\mathrm{mm} \times 2.1 \mathrm{~mm} \times 3 \mathrm{~mm}$; Restek, Bellefonte, PA, USA) column was selected for chromatographic separations. MS/MS detection was performed using electrospray negative and positive ionization in MRM mode, and injection volumes were 10 and $20 \mu \mathrm{L}$, respectively. A fully validated, qualitative LC-HRMS method with the ability to detect over 130 NPS with their metabolites was used. Validation was performed following the SWGTOX guidelines [13]. The limit of detection (LOD) values for all target analytes were in the ranges of 0.02 and $0.8 \mathrm{ng} / \mathrm{mL}$. LC-HRMS analyses were carried out using an HR Q-Exactive Plus Mass Spectrometer equipped with a Dionex Ultimate 3000 RS UHPLC System and an Agilent Poroshell 120 EC-C18 column (2.7 $\mathrm{mm} \times 150 \mathrm{~mm} \times 4.6 \mathrm{~mm}$; Agilent, Santa Clara, CA, USA). Full MS scan data were obtained from m/z 120 to 650 at a resolution of 35,000, and data-dependent MS/MS spectra were obtained at a resolution of 17,500 by using stepped normalized collision energy (NCE), 17.5, 35, and 52.5\%, with Top-7 MS/MS experiments. The injection volume was $10 \mu \mathrm{L}$. The SPSS software (IBM, Armonk, NY, USA) was used for the statistical analysis, which included performing the chi-square test and Poisson regression analysis with the probability level set to $P<0.05$.

\section{Results}

In the present study, suspected drug abuse cases were screened during the first wave of the COVID-19 pandemic in Turkey. Home confinement lasted from March 13 to May 31, 2020. Four toxicological control measurements were obtained quarterly between March 2019 and February 2020, corresponding to the phase before the lockdown. Furthermore, four more toxicological controls were obtained quarterly between March 2020 and February 2021 that included the lockdown period, and the last three measurements, which concerned the postlockdown quarter, were obtained quarterly between June 2020 and February 2021 (in Fig. 1, 
Fig. 1 Timeline of the COVID19 pandemic in Turkey and sample collection periods

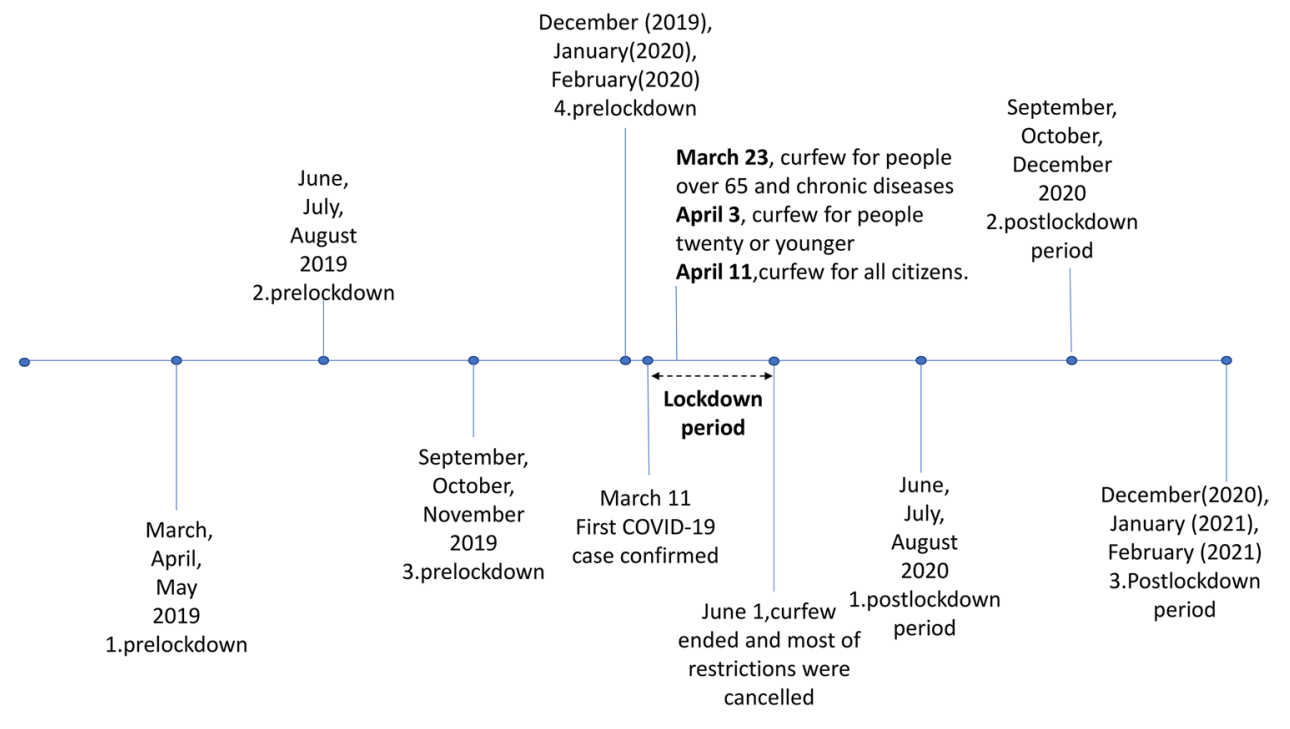

timeline of the COVID-19 pandemic in Turkey and sample collection periods is shown).

In the present study, 8727 drug abuse suspect samples were analyzed during the investigation period before COVID-19 (March 1, 2019 to February 29, 2020), and 9669 suspects were analyzed quarterly during the first wave of the COVID-19 pandemic in Turkey during the same investigation period in 2020 (March 1, 2020 to February 28, 2021). The distribution by gender was $8.3 \%$ female and $92.7 \%$ male in 2019 and $12.6 \%$ female and $87.4 \%$ male in 2020 . The numbers of female drug abuse suspects increased significantly in $2020(p<0.001)$. Among young adults between the ages of 18 and 24, drug use was significantly higher than that in the previous year $(p<0.05)$. The distribution among other age categories were similar in the two investigation periods $(p>0.05)$ (in Table 1, the distribution of positive cases is given).

The percentage of positive samples was 63.7\% $(n=5563)$ in 2019 and $69.3 \%(n=6,702)$ in 2020 (Fig. 2). Two or more substances were detected in $73.7 \%(n=4,099)$ of the cases in 2019 and in $89.3 \%(n=5985)$ of the cases in 2020.

The prevalence of classical illicit drugs (MDMA, amphetamine, methamphetamine, cocaine, heroin, cannabis, and methadone) was analyzed for 2019 and 2020 during the investigation period (Table 2). The frequencies of amphetamine, methadone, and heroin consumption were similar in the two investigation periods $(p>0.05)$. Heroin consumption could be proved in cases as the samples were also positive for 6-acetyl morphine (6AM) and not only for morphine and codeine. Additionally, cocaine and cannabis consumption could be proved in cases as the samples were also positive for their metabolites (THC-COOH, benzoylecgonine, and/or ecgonine methyl ester), not only for cocaine and THC. MDMA, cannabis, and cocaine use decreased significantly in 2020 ( $p<0.001)$ (Fig. 2). MDMA-positive cases
Table 1 Distribution of the positive cases according to gender and age

\begin{tabular}{llll}
\hline & 2019 & 2020 & $X^{2}(P)$ \\
\hline Female & $462(8.3 \%)$ & $843(12.6 \%)$ & $* 58.388(0.001)$ \\
Male & $5101(92.7 \%)$ & $5859(87.4 \%)$ & \\
Total number & $5563(100 \%)$ & $6702(100 \%)$ &
\end{tabular}

Age distribution of the positive cases

\begin{tabular}{llll}
$\begin{array}{l}\text { Age category } \\
<18\end{array}$ & $651(11.7 \%)$ & $744(11.1 \%)$ & $1.090(0.297)$ \\
$18-24$ & $3621(65.1 \%)$ & $4510(67.3 \%)$ & $* 6.600(0.01)$ \\
$25-29$ & $668(12.0 \%)$ & $764(11.4 \%)$ & $1.091(0.296)$ \\
$30-39$ & $545(9.8 \%)$ & $596(8.9 \%)$ & $2.944(0.086)$ \\
$40-44$ & $51(0.9 \%)$ & $54(0.8 \%)$ & $0.442(0.506)$ \\
$\geq 45$ & $27(0.5 \%)$ & $34(0.5 \%)$ & $0.030(0.863)$ \\
Total number & 5563 & 6702 & \\
\hline
\end{tabular}

$x^{2}$ and $P$ values are shown for Pearson $\mathrm{x}^{2}$ statistical analysis between the 2 years. $* P<0.05$

decreased from 887 (2019) to 530 (2020). Cannabis-positive cases decreased from 2843 (2019) to 2189 (2020). Similarly, cocaine-positive cases decreased from 490 (2019) to 417 (2020). Methamphetamine use was significantly higher than that in the previous year $(p<0.001)$, and the number of positive cases increased from 726 (2019) to 2151 (2020).

Only synthetic cannabinoids were present in the samples, and no other psychoactive substances were detected in the 2 years. The prevalence of synthetic cannabinoid abuse was similar in the 2 years, but some differences were observed in substances. Synthetic cannabinoids were detected (> $47 \%$ of positive cases), including 4F-MDMB-BUTINACA (at a frequency of $28.0 \%$ ), 5F-MDMB-PICA (62.2\%), MDMB-4en-PINACA (4.5\%), 5F-ADB (4.1\%), and ADBFUBINACA (1.2\%), in 2019. The frequency of synthetic 
Fig. 2 Number and names of positive drugs during the same selected periods a in 2019 and b 2020
(a)
2019

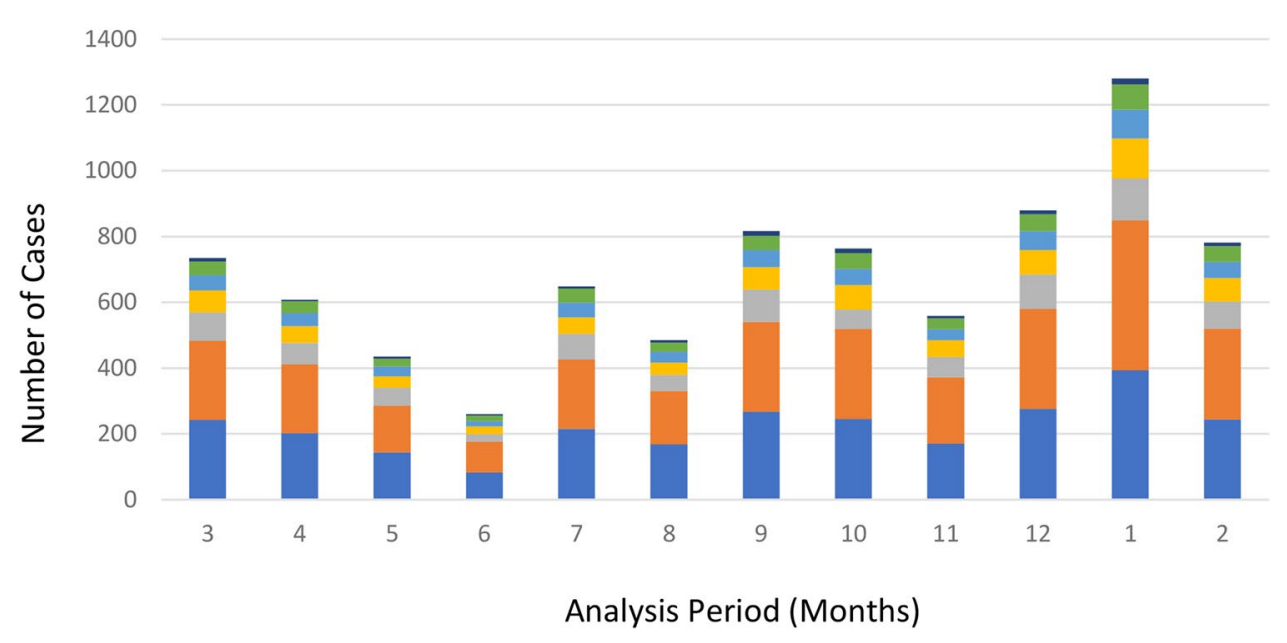

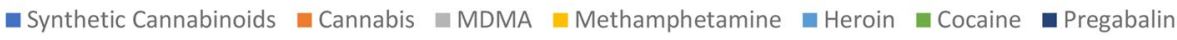

2020

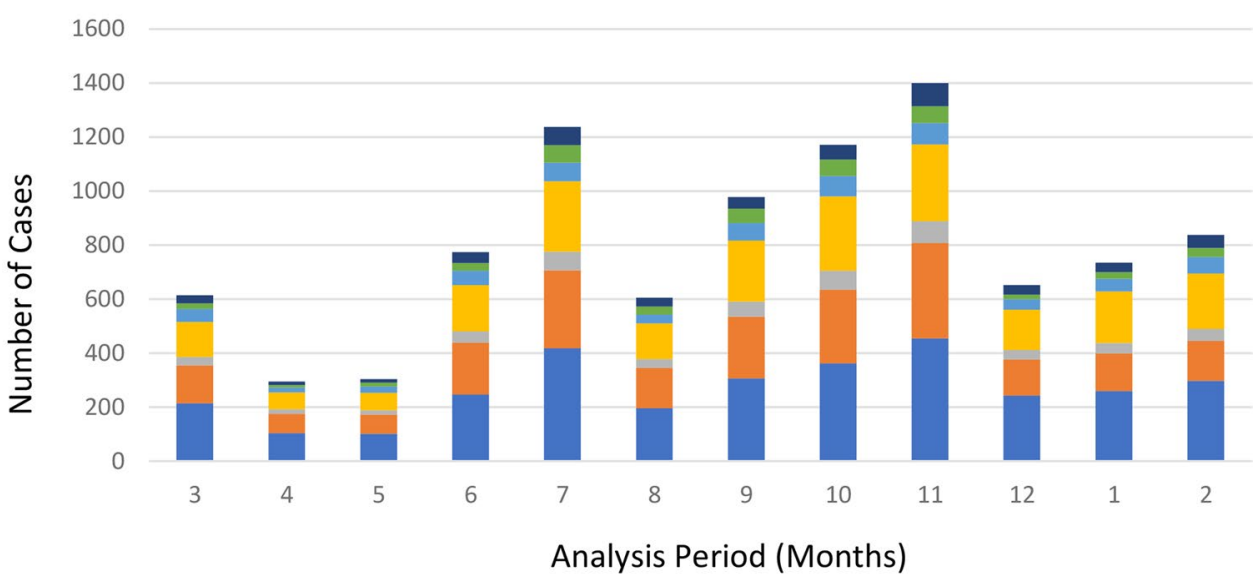

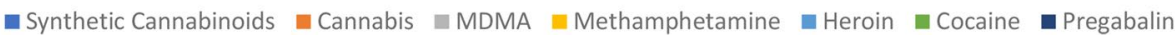

cannabinoids detected ( $>47 \%$ of positive cases) was 4F-MDMB-BUTINACA (0.1\%), 5F-MDMB-PICA (23.0\%), and MDMB-4en-PINACA (76.3\%) in 2020. The abundances of 4F-MDMB-BUTINACA and 5F-MDMB-PICA decreased significantly in 2020, and the abundances of MDMB-4enPINACA increased. 5F-ADB and ADB-FUBINACA were not detected in 2020. These can be explained by changing patterns of distribution in the black market and changes in supply. In the majority of positive cases, synthetic cannabinoids were present in combination with methamphetamine, cannabis, MDMA, cocaine, heroin, or pregabalin.

Among the investigated licit drugs (Table 2), pregabalin was the most abused licit drug in the group. Additionally, diazepam and clonazepam were limited but present in both years. Diazepam and clonazepam consumption was similar in the 2 years $(p>0.05)$. Pregabalin use increased significantly in $2020(p<0.001)$, and the number of positive cases increased from 115 (2019) to 497 (2020). Tramadol and buprenorphine consumption was similar in the 2 years $(p$ $>0.05)$. Ketamine consumption was detected only in 2020 $(n=6$; Table 2).

In total, 1330 cases were analyzed during the lockdown period (March 2020 and May 2020), 2082 suspects were analyzed in the same period in the prelockdown period (March 2019 and May 2019), and the percentages of positive samples were $57.1 \%$ and $64.9 \%$, respectively. In the first postlockdown period (June 2020 and August 2020), 2717 cases were analyzed, 1488 cases were analyzed in the same 
Table 2 Frequency of illicit and licit drugs in all positive samples

\begin{tabular}{|c|c|c|c|c|c|}
\hline & \multicolumn{2}{|l|}{2019} & \multicolumn{2}{|l|}{2020} & \multirow[b]{2}{*}{$X^{2}(P)$} \\
\hline & Positive cases ${ }^{\mathrm{a}}$ & In comb. (\%) & 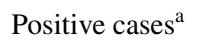 & In comb. $(\%)$ & \\
\hline \multicolumn{6}{|l|}{ "İllicit" drugs } \\
\hline S. cannabinoids & $2653(47.7 \%)$ & 64.7 & $3201(47.8 \%)$ & 58.9 & $0.006(0.937)$ \\
\hline MDMA & $887(15.9 \%)$ & 72.8 & $530(7.9 \%)$ & 72.3 & *192.13 (0.001) \\
\hline Methamphetamine & $726(13.1 \%)$ & 56.6 & $2151(32.1 \%)$ & 73.8 & *614.05 (0.001) \\
\hline Amphetamine & $28(0.5 \%)$ & 30.8 & $31(0.5 \%)$ & 89.4 & $0.11(0.745)$ \\
\hline THC-COOH & $2843(51.1 \%)$ & 33.9 & $2189(32.7 \%)$ & 43.5 & *427.38 (0.001) \\
\hline Cocaine & $490(8.8 \%)$ & 67.0 & $417(6.2 \%)$ & 67.7 & $* 29.69(0.001)$ \\
\hline Heroin & $537(9.7 \%)$ & 35.4 & $628(9.3 \%)$ & 58.2 & $0.28(0.595)$ \\
\hline Methadone & $87(1.6 \%)$ & 87.7 & $102(1.5 \%)$ & 94.6 & $0.035(0.851)$ \\
\hline \multicolumn{6}{|l|}{ "Licit" drugs } \\
\hline Tramadol & $26(0.5 \%)$ & 13.0 & $40(0.6 \%)$ & 82.4 & $0.96(0.328)$ \\
\hline Buprenorphine & $14(0.2 \%)$ & 86.7 & $17(0.2 \%)$ & 100.0 & $0.01(0.977)$ \\
\hline Clonazepam & $25(0.5 \%)$ & 67.4 & $44(0.7 \%)$ & 78.0 & $2.33(0.127)$ \\
\hline Pregabalin & $115(2.1 \%)$ & 45.8 & $497(7.4 \%)$ & 75,3 & *183.422 (0.001) \\
\hline Diazepam & $36(0.7 \%)$ & 60.9 & $42(0.6 \%)$ & 68,9 & $0.020(0.887)$ \\
\hline Ketamine & 0 & & $6(0.1 \%)$ & 83.3 & \\
\hline
\end{tabular}

${ }^{a}$ The percentage of positive cases are in relation to the total number of positive cases. In combination (\%): number of positive cases for a drug in combination with other drugs/total number of positive cases for the respective drug $\times 100 . x^{2}$ and $P$ values are shown for Pearson $x^{2}$ statistical analysis between the 2 years. ${ }^{*} P$ $<0.05$

period in the prelockdown period (June 2019 and August 2019), and the percentages of positive samples were $67.1 \%$ and $62.2 \%$, respectively. In the second postlockdown period (September 2020 and November 2020), 3426 cases were analyzed, while 2288 cases were analyzed over the same length of time in the prelockdown period (June 2019 and August 2019); the percentages of positive samples found in these analyses were $70.9 \%$ and $65.9 \%$, respectively. In the last postlockdown period (December 2020 and February 2021), 2196 cases were analyzed over the same length of time in the last prelockdown period (December 2019 and February 2020); the percentages of positive samples found in these analyses were $72.0 \%$ and $67.2 \%$, respectively. Poisson regression analysis for drug frequency over time showed no significant changes during the investigation periods. However, comparisons between COVID-19 affected and nonaffected drug abuse suspects may be confounded by unmeasured differences in the two populations [9].

\section{Discussion}

COVID-19, the largest public health crisis of our time, has caused global concern throughout the world over the past year and has led to several challenges in various ways. The primary measures to contain the pandemic, such as home confinement and sustained lockdown, have led to overwhelming economic burden at the community level and forced the community to face various undesirable emotional reactions, psychological difficulties, and behavioral changes, including excessive substance abuse [14]. Coronavirus is also causing hidden challenges to health care and wider social structures. Among the vulnerable populations are persons who smoke or vape, use opioids, or have a substance use disorder (SUD). Because of impediments to delivering care to this population, persons with SUD who develop COVID-19 may find it harder to receive care. Those in recovery are also uniquely challenged by social distancing measures [15].

The patterns of illicit and licit drug consumption among suspected drug users were similar in 2019 and 2020, but some differences were observed. In 2019, ketamine was not detected during the investigation period. The results provide excellent data to characterize the distribution of abusive illicit and licit drugs among suspected drug user populations during the investigation period of 2019 and 2020 . The frequency of positive cases was approximately $60-70 \%$ in the 2 years in the suspected drug user population. Positive cases among suspected drug users were found at a high frequency. In addition, the combined use of illicit drugs increased significantly in $2020(p<0.001)$. The female gender and/or young age user showed significant increases in 2020. This distribution pattern of users may be associated with more negative psychological effects of COVID-19 and higher levels of stress, anxiety, and depression among corresponding individuals [14]. 
Pubs and night clubs have been closed, concerts and festivals have been canceled, and the movements of people and social interactions have been strictly restricted by the implementation of quarantine and lockdown preventions [7]. The use of illicit drugs MDMA and cocaine, which are linked to night life and parties, was expected to decrease because of these precautions [16]. Similarly, the results from the present study show a significant decrease in MDMA and cocaine use during the lockdown, and it did not tend to return to prelockdown levels in the postlockdown period because parties continued to be canceled in the postlockdown period. Strict controls at customs and border crossings would reduce the availability of cocaine and MDMA at the market. Small and cautious home parties instead of large and imprudent parties would reduce the probability of being caught by the police.

Our data also show a reduction in cannabis use during the lockdown, and it did not rise back to prelockdown levels in the postlockdown period. This pattern may be partly explained by difficulties in accessing cannabis, indicative of localized supply shortages, and a considerable increase in prices [17]. There was a statistically significant decrease in MDMA and cocaine use, and a slight decrease was reported for cannabis use during the lockdown $[16,17]$. In contrast to MDMA, cocaine, and cannabis, the study showed a significant increase in methamphetamine use during the lockdown, and it did not tend to return to prelockdown levels in the postlockdown period. Despite supply shortages in other illicit drugs, methamphetamine was illegally transported between Turkish-Iranian provinces bordering rural mountainous areas, where there are no formal border crossing points [18]. The Turkish Narcotic Police Department seized the largest amount of methamphetamine in Turkish history [19]. The ease of availability of methamphetamine at the black market compared to other drugs increased the frequency of abuse. With respect to the increase of methamphetamine consumption, a stimulant methamphetamine might have been found more helpful for situations like "unemployment," "critical financial problems," "personal problems," and "home schooling."

In addition to methamphetamine, pregabalin use increased significantly as a licit drug. The deficiency of classic illicit drugs, together with the difficulty of going out to look for them, might have induced addicts to misuse or illegal use of licit drugs with prescriptions, such as pregabalin.

The analysis of suspected drug abuse cases showed an overall significant reduction in the number of suspected cases during the lockdown period (March 2020 and May 2020) compared to the same period in the prelockdown period (March 2019 and May 2019) $(p<0.001)$, but the percentage of positive cases increased from 57.1 to $64.9 \%$. Because of strictly reduced movement due to restrictions and a total lockdown, suspected drug users may not have been caught by routine police search. In the following first quarter
(June 2020 and August 2020) after lockdown with reduced restrictions, the number of suspected cases increased significantly compared to the same periods in the prelockdown period (June 2019 and August 2019) $(p<0.002)$, and the percentage of positive cases increased from 62.2 to $67.1 \%$. In the second postlockdown period (September 2020 and November 2020), a significant increase continued compared to the same periods in the prelockdown period (September 2019 and November 2019) $(p<0.001)$, and the percentage of positive cases increased from 65.9 to $70.9 \%$. In the last postlockdown period (December 2020 and February 2021), the increase continued compared to the same periods in the prelockdown period (November 2019 and February 2020), and the percentage of positive cases increased from 67.6 to $72.0 \%$. The results of this study show an overall change in the pattern of drug use and percentages as a result of the phenomenal case related to COVID-19. Eventually, the COVID-19 pandemic could lead to a spike in drug abuse in suspected drug users, new trends in illicit drugs, and potentially the development of addiction in at-risk individuals, thereby placing further pressure on drug addiction services and health care services during the postpandemic period.

\section{Conclusion}

COVID-19 has caused a turmoil effect on the economy, tourism, community life, healthcare, education, sport, and finance. The effects of the COVID-19 pandemic on daily life, health, and society are not yet clearly known. The restrictions that were applied to control the pandemic and lockdown affected drug abuse and the trends of abuse. The study found a significant increase in drug abuse and a change in the frequency of abused drugs among suspected drug users. In particular, there was an elevated trend to replace less harmful and habitual drugs with other potentially dangerous but more easily available substances, such as methamphetamine and pregabalin. This increase in the use of abused drugs and combined drug use trends has continued after the lockdown, leading to an elevated risk of developing new addictions and other elevated health risk problems. The comprehensive data show the drug abuse trends and drug use patterns in Turkey before the pandemic, during the lockdown period and after the lockdown period among suspected drug users. The study will be useful to understand the effect of COVID-19 on drug abuse frequencies and trends in Turkey. It would also be useful to combine experimental data about the consumption of drugs with other data collected during the COVID-19 pandemic in other countries.

Supplementary Information The online version contains supplementary material available at https://doi.org/10.1007/s00414-022-02794-1. 


\section{Declarations}

Ethics approval This study was conducted with the approval of the Forensic Medicine Council Scientific Board of Ministry of Justice Turkey. All procedures performed in this study were in accordance with the ethical standards of the national committee and with the 1964 Declaration of Helsinki. Biological fluid (blood and urine) collection from drug use suspects was conducted by judicial authorities, and the samples were sent to the Forensic Medicine Council for analysis at their request. Blank blood samples were obtained from a regional blood donation center, and blank urine samples were collected from healthy nondrug user volunteers.

Conflict of interest The authors declare no competing interests.

\section{References}

1. Huang C, Wang Y, Li X, Ren L, Zhao J, Hu Y, Zhang L, Fan G, Xu J, Gu X, Cheng Z, Yu T, Xia J, Wei Y, Wu W, Xie X, Yin W, Li H, Liu M et al (2020) Clinical features of patients infected with 2019 novel coronavirus in Wuhan, China. Lancet 395:497-506. https://doi.org/10.1016/S0140-6736(20)30183-5

2. Coronavirus Disease (COVID-19) events as they happen World Health Organization (2020). https://www.who.int/emergencies/ diseases/novel-coron avirus-2019/events-as-they-happen. Accessed 9 March 2021

3. Covidvisualizer. (2021) COVID-19 visualizer. https://www.covid visualizer.com. Accessed 23 September 2021

4. COVID-19 web page of the Republic of Turkey (2021). Ministry of Health. Available Ministry of Health, Republic of Turkey https://covid19.saglik.gov.tr. Accessed 13 May 2021

5. COVID-19 pandemic in Turkey (2021). Wikipedia https://en. wikipedia.org/wiki/COVID-19_pandemic_in_Turkey. Accessed 3 May 2021

6. Ahorsu DK, Lin CY, Imani V, Saffari M, Griffiths MD, Pakpour AH (2020) The fear of COVID-19 scale: development and initial validation. Int J Ment Heal Addict 27:1-9. https://doi.org/10.1007/ s11469-020-00270-8

7. Mental health and psychosocial considerations during the COVID19 outbreak (2020). World Health Organization (WHO). https:// apps.who.int/iris/bitstream/handle/10665/331490/WHO-2019nCoV-MentalHealth-2020.1-eng.pdf. Accessed 2 May 2021

8. Volkow ND (2020) Collision of the COVID-19 and addiction epidemics. Ann Intern Med 173:61-62. https://doi.org/10.7326/ M20-1212

9. Goldmann E, Galea S (2014) Mental health consequences of disasters. Annu Rev Public Health 35:169-183. https://doi.org/10. 1146/annurev-publhealth-032013-182435
10. Öztürk YE, Yeter O, Öztürk S, Karakus G, Ates I, Buyuk Y, Yurdun $T$ (2018) Detection of metabolites of the new synthetic cannabinoid CUMYL-4CN-BINACA in authentic urine samples and human liver microsomes using high-resolution mass spectrometry. Drug Test Anal 10:449-459. https://doi.org/10.1002/dta.2248

11. Yeter O, Erol Öztürk Y (2019) Detection and quantification of 5F-ADB and its methyl ester hydrolysis metabolite in fatal intoxication cases by liquid chromatography-high resolution mass spectrometry. Forensic Sci Int 302:109866. https://doi.org/10.1016/j. forsciint.2019.06.024

12. .Turkish Criminal Law, Number: TCK-191 (2021). Ministry of Justice. https://www.mevzuat.gov.tr/MevzuatMetin/1.5.5237.pdf . Accessed 13 September 2021

13. Scientific Working Group for Forensic Toxicology (SWGTOX) (2013) Standard practices for method validation in forensic toxicology. J Anal Toxicol 37:452-474. https://doi.org/10.1093/jat/ bkt054

14. Dubey S, Biswas P, Ghosh R, Chatterjee S, Dubey MJ, Chatterjee S, Lahiri D, Lavie CJ (2020) Psychosocial impact of COVID-19. Diabetes Metab Syndr Clin Res Rev 14:779-788. https://doi.org/ 10.1016/j.dsx.2020.05.035

15. Gili A, Bacci M, Aroni K, Nicoletti A, Gambelunghe A, Mercurio I, Gambelunghe $C$ (2021) Changes in drug use patterns during the COVID-19 pandemic in Italy: monitoring a vulnerable group by hair analysis. Int J Environ Res Public Health 18:1-11. https://doi. org/10.3390/ijerph18041967

16. Impact of COVID-19 on drug markets, use, harms and drug services in the community and prisons (2021). European Monitoring Centre for Drugs and Drug Addiction (EMCDDA). https://www. emcdda.europa.eu/system/files/publications/13745/TD0321143E NN_002.pdf19.4. Accessed 21 April 2021

17. Vanderbruggen N, Matthys F, Van Laere S, Zeeuws D, Santermans L, Van Den Ameele S, Crunelle CL (2020) Self-reported alcohol, tobacco, and cannabis use during COVID-19 lockdown measures: results from a web-based survey. Eur Addict Res 26:309-315. https://doi.org/10.1159/000510822

18. Ekici B, Ozbay S (2013) Iranian methamphetamine and Turkey: an emerging transnational threat. Trends Organ Crime 16:286-305. https://doi.org/10.1007/s12117-013-9204-6

19. Turkey drug report. (2020) General Directorate of Security. http://www.narkotik.pol.tr/kurumlar/narkotik.pol.tr/TUB\%C4\% B0M/TU\%CC\%88RKI\%CC\%87YE-UYUS\%CC\%A7TURUCURAPORU_26-HAZI\%CC\%87RAN-FI\%CC\%87NAL-_1_.pdf. Accessed 13 March 2021

Publisher's note Springer Nature remains neutral with regard to jurisdictional claims in published maps and institutional affiliations. 